\title{
Resumen
}

Los procesos para proyectar y modelar la actividad económica en Puerto Rico son fundamentales en la toma de decisiones tanto para el sector público, como para el sector privado. No llevar a cabo este proceso en la forma correcta puede acarrear costos altos, los cuales se reflejan en ineficiencias en los procesos de decisión. En este trabajo se presenta un método alterno para modelar la actividad económica, basado en la teoría económica y la información estadística y matemática de las series a estudiar.

Palabras clave: Proyecciones, métodos matemáticos y estadísticos

\begin{abstract}
Forecasting and modeling economic activity in Puerto Rico, is fundamental to efficient and effective decision-making processes. Consequently, the failure to do so in Puerto Rico, in both the public and private sectors, bares high costs wich can be observed in the inefficiencies of each sector decision making. Based in economic theory as well as statistical and mathematical information processes, this paper presents an alternative method for modeling economic activity.
\end{abstract}

Key words: Forecast, mathematical and statistical methods 


\title{
Dr. Carlos A. Rodríguez*
}

\section{Una nueva propuesta para modelar y proyectar la actividad económica en Puerto Rico}

\author{
"Las definiciones y procedimientos formales \\ surgen de la investigación de problemas prácti- \\ cos." \\ El método de Arquímides
}

\section{Introducción}

ctualmente, los modelos econométricos principales utiliza-
dos en Puerto Rico, para analizar la estructura de la Isla y
proyectar las variables macroeconómicas principales se basan en sistemas de ecuaciones simultáneas. Como método de estimación se utiliza la técnica de mínimos cuadrados ordinarios. Sin embargo, casi todos los años estos modelos son criticados y, por ende, las proyecciones a corto y largo plazo de las variables macroeconómicas principales en la Isla. Las críticas van desde las consideraciones econométricas de los modelos y los efectos de la política pública en los parámetros del modelo, hasta la sobrestimación del crecimiento del Producto Nacional Bruto Real.

* Catedrático Auxiliar de Economía, Departamento de Economía, Facultad de Ciencias Sociales, Universidad de Puerto Rico, Recinto de Río Piedras, PR. Recibido: 15 de enero de 2004; Aceptado: 13 de agosto de 2004. 
Además, no se consideran aspectos importantes como la estabilidad de los parámetros a través del tiempo para así, por ejemplo, refutar la crítica de Lucas (Lucas, 1972, Lucas y Sargent, 1975), los supuestos de exogeneidad de las variables consideradas para hacer restricciones en los modelos, así como el orden de integración de las series, la corroboración de la existencia de relaciones a largo plazo, las estructuras de rezago y la dinámica del modelo (Eatwell, Milligate y Newman, 1990; Ericsson y Irons 1994; Banerjee, Dolado, Galbraith, y Hendry, 1993; Sims, 1975, 1986).

Cabe señalar que la utilización de modelos de ecuaciones simultáneas tiene una gran tradición en la econometría, en particular la tuvo durante las décadas de 1940 y 1950 (Galindo, 1995, 1997; Hendry, 1997; Spanos, 1986). Para ese tiempo, lo que se buscaba era crear un modelo que fuese tan preciso que pudiera responder ante cualquier eventualidad de índole económico y que tuviese la capacidad de analizar de manera precisa la evolución de las series macroeconómicas (Lucas y Sargent, 1975). El análisis se basaba en lograr el mejor ajuste estadístico. En particular, se intentaba que tanto la media como la varianza del término de error de las diversas ecuaciones econométricas en el sistema no mostrasen ninguna tendencia. De acuerdo al comportamiento del término de error en el modelo es que pude indicarse si un modelo puede ser utilizado o no (Maddala, 1996).

Sin embargo, a partir de la década de 1970 el uso de estos modelos ha sido muy criticado, en especial en el proceso de toma de decisiones de política pública. A raíz de lo anterior, se desarrollaron una serie de modelos uniecuacionales y multiecuacionales los cuales intentan resolver los problemas presentados por los modelos de ecuaciones simultáneas (Pankratz, 1995; Galindo, 1997; Sims, 1975, 1980, 1987, 1987; Enders, 1995; Sims, y Zha, 1998). Estos métodos de modelación matemática y econométrica forjan la base de la econometría moderna.

La econometría moderna puede definirse como el estudio sistemático de los fenómenos económicos utilizando la información teórica y empírica (Spanos, 1986, Galindo, 1995). Además, estudia estos fenómenos mediante la aplicación de métodos estadísticos a los datos observados. 
Dado lo anterior, en este trabajo se propone un método nuevo, para proyectar y simular la actividad económica en Puerto Rico, en función de la elaboración de un marco conceptual general que incluye diversas metodologías de estimación como opciones particulares. ${ }^{1}$ Las diversas visiones metodológicas pueden ordenarse de acuerdo a las siguientes dos líneas estratégicas (Galindo, 1995):

i. Una posición epistemológica en cuanto al uso de métodos estadísticos en una ciencia no experimental;

ii. Una posición sobre la patología de los estimadores y las pruebas econométricas a utilizar.

Para desarrollar este trabajo, en la segunda parte, se hace una comparación en cuanto a los supuestos de la econometría tradicional y la moderna, haciendo énfasis en el supuesto de especificación correcta y en la determinación del comportamiento del término de error en ambos métodos. En la tercera parte se presenta el desarrollo de la econometría moderna, la cual se propone como una alternativa a los problemas que presentan los modelos actuales. Por último, se presentan los comentarios generales.

\section{El método tradicional y el moderno}

Para generar cualquier tipo de modelo econométrico, hay que tener en cuenta que en un sistema económico existen un sinnúmero de interrelaciones complejas no lineales, interdependientes y multivariadas en las cuales los agentes intentan tanto optimizar sujeto a sus restricciones, como adaptar su comportamiento a situaciones cambiantes, donde la incertidumbre desempeña un papel fundamental. Por lo tanto, existen diversos procesos de ajuste dinámico a los errores pasados, formación de expectativas y choques aleatorios que condicionan el comportamiento económico general.

El uso de métodos estadísticos en una ciencia, en principio, no experimental puede justificarse utilizando los siguientes dos enfoques: 
1. La teoría econométrica tradicional, la cual es la que se utiliza actualmente en Puerto Rico, asume el supuesto de especificación correcta, de tal manera que el uso de la estadística es justificado al incluir una ecuación a estimar el término de error con ciertas propiedades estadísticas. Esto permite el uso de la inferencia estadística y está sujeta a la crítica de Sims (1980) en cuanto a la inclusión arbitraria de restricciones en los parámetros.

2. La econometría moderna se asume como una ciencia no probabilística con errores de observación. Esto significa la adopción de un acercamiento probabilístico como el fundamento de la econometría. Lo anterior se origina con la conocida revolución de Havelmo. En este caso, el concepto de incertidumbre en los datos permite pasar de una ciencia determinística a una teoría estocástica.

La primera justificación, la tradicional, señala que dado el supuesto de especificación correcta, no existe un sesgo de especificación en el modelo econométrico utilizado. Si este supuesto se cumple, entonces el valor esperado del término error es igual a cero, el modelo es homocedástico, no hay autocorrelación y la covarianza entre el término de error y la(s) variable(s) independientes es igual a cero. En este caso, el término de error es una entidad independiente que permite incluir ciertas propiedades estocásticas a un modelo. Aspecto que justifica la búsqueda del mejor ajuste estadístico. Es decir, si el modelo cumple con los supuestos establecidos respecto al término de error entonces puede ser utilizado.

De acuerdo con la segunda justificación, la econometría moderna estudia de manera sistemática, mediante la información empírica y teórica, estos fenómenos considerando que (Galindo, 1995):

i. La información empírica y la teórica tienen igual importancia;

ii. Existe un proceso generador de información (PGI) el cual no es observable, pero se infiere a través del análisis de las características estadísticas y probabilísticas de los datos. 
En este caso, la econometría moderna utiliza la inferencia estadística con datos no experimentales dado el supuesto de la existencia de un proceso generador de información (PGI). Los datos son considerados como una posible realización de este PGI, el cual se compone de una secuencia de vectores aleatorios (Spanos, 1986). Las series económicas pueden ser modeladas a partir de una distribución conjunta de probabilidades de las variables incluyendo el tiempo (Galindo, 1995). Dichas variables se asumen como independientes e idénticamente distribuidas. Por lo que, a diferencia de la econometría tradicional, en la econometría moderna, el término de error es sólo una entidad derivada de las propiedades estadísticas de las series.

\section{La econometría moderna: una nueva alternativa}

El objetivo de la econometría moderna es el de especificar y estimar un modelo estadístico que represente una aproximación adecuada del PGI. Por lo que hay que considerar los siguientes puntos que son fundamentales a la hora de generar un modelo econométrico (Galindo, 1995; Hendry, 1995):

1. La información teórica se encuentra, normalmente, sintetizada en una formalización matemática conocida como el modelo teórico. Los conceptos teóricos no coinciden, por lo general, con la información estadística disponible,

-El modelo teórico debe transformarse en uno estimable, el cual es especificado en cuanto a las variables observadas.

* Pueden existir diversas versiones estimables de un modelo teórico

-Esto explica la existencia, en muchos casos, de evidencia contradictoria sobre un modelo teórico.

2. La información empírica se resume en las propiedades estadísticas capturadas en los modelos probabilístico y muestral,

3. El mecanismo estadístico general (MEG) es una formalización probabilística que contiene la información muestral elaborada para 
analizar el modelo a estimar y representa una primera aproximación del PGI. El MEG está compuesto por el modelo probabilístico y el modelo muestral. El MEG tiene las siguientes características (Galindo, 1995; Spanos 1986; Hendry, 1995; Hendry, 1997):

1. Es un modelo en su forma reducida sin incluir ninguna restricción en los parámetros.

2. Postula una combinación consistente entre la especificación teórica inicial, que representa ya una selección de variables relevantes y la proposición acerca de la estructura estocástica del proceso.

3. La inferencia estadística se realiza a través del estudio de las propiedades de la distribución conjunta de las variables aleatorias.

-Postula un determinado proceso estocástico como una aproximación del PGI.

4. Transforma la incertidumbre acerca del PGI en la incertidumbre de la estimación de unos parámetros desconocidos.

-Incluye la noción de probabilidad e incertidumbre en el modelo econométrico mediante la inclusión de un conjunto de parámetros desconocidos de una familia de funciones de densidad de probabilidad.

5. Es definido directamente en cuanto a las variables aleatorias y no solamente respecto al término de error.

-Las propiedades estadísticas del término de error se derivan naturalmente de las propiedades estocásticas de las series económicas.

6. Del MEG se obtiene un modelo econométrico mediante diferentes reparametrizaciones, transformaciones y reducciones del espacio paramétrico.

En términos generales, el PGI puede representarse como un proceso estocástico con una distribución probabilística infinita:

(1) $F\left(X_{t 1}, X_{t 2}, \ldots, X_{t n}\right)$ 
En esta ecuación cada $\mathrm{X}_{\mathrm{ti}}$ representa una variable aleatoria en el periodo t. Por cuestiones operacionales debe reducirse el número de parámetros desconocidos, los cuales definen al proceso estadístico. Esto se realiza utilizando la teoría y la información provista por los datos. La importancia que se le dé a cada variable conduce a diversas formas de modelaje econométrico. No obstante, la reducción de parámetros se realiza siguiendo los siguientes puntos (Galindo, 1995):

1. La información teórica y la reducción del número de parámetrosLa teoría económica sugiere una selección de variables relevantes sobre un fenómeno económico dado. Esta información dada por la teoría se incorpora en el modelo estadístico mediante la reducción del número de variables en consideración y/o condicionando el valor de algunos parámetros. Por lo tanto, la teoría económica proporciona argumentos necesarios, los cuales ayudan a definir la distribución condicional del fenómeno económico que se esté estudiando y reduce el espacio paramétrico indicando cuales son las funciones de probabilidad irrelevantes. Quiere decir que un mejor conocimiento de la teoría implica una mejor especificación del modelo condicional y una reducción en el número de parámetros a estimar, aumentando así la eficiencia de las estimaciones.

2. La información teórica y la reducción de parámetros - También puede utilizarse la información empírica para reducir el espacio paramétrico. Sin embargo, esto sucede cuando el proceso estocástico satisface las siguientes condiciones:

a. Simetría:

(2) $F\left(X_{t i}, \ldots, X_{t n}\right)=F\left(X_{t j} \ldots, X_{t m}\right)$, donde j y m representan cualquier permutación de i y $\mathrm{n}$.

b. Compatibilidad:

(3) $\operatorname{LimE}\left(X_{t b}, \ldots, X_{t n}\right)=F\left(X_{t b} \ldots, X_{t m-1}\right)$

$\mathrm{X} \infty$ 
Un proceso estocástico que cumple con las condiciones de simetría y compatibilidad, puede reducirse al imponerse determinadas restricciones en los parámetros desconocidos, de tal manera que las series económicas puedan representarse como un proceso m-dependiente (Spanos, 1986). Las restricciones más comunes son las siguientes (Spanos, 1986; Galindo, 1995):

1. Restricción de heterogeneidad del proceso en el tiempo ${ }^{2}-\mathrm{Al}$ ser el proceso estocástico definido:

(4) $F\left(X_{t b}, \ldots, X_{t n}\right)=F\left(X_{t+n}, \ldots, X_{t+n+m}\right)$,

El proceso estocástico es estrictamente estacionario, ya que su función de distribución no cambia con el tiempo, por lo que su media y varianza se mantienen constantes a través del tiempo. En un proceso estacionario los vectores aleatorios se distribuyen idénticamente con homogeneidad en el tiempo;

2. Restricción en la memoria del tiempo - En esta restricción las relaciones entre $\mathrm{X}_{\mathrm{ti}} \mathrm{y}_{\mathrm{tj}}$ disminuyen a medida que la diferencia entre i y j aumenta. Esto se observa en las series cuando el proceso estocástico es asintóticamente no-correlacionado y la función de autocorrelación tiende a cero a medida que la distancia entre i y j aumenta.

\section{PGI y MEG}

Según la sección anterior, la reducción de parámetros utilizando la teoría económica o las propiedades de las series son las herramientas esenciales para que el MEG sea una representación adecuada del PGI. Por lo tanto, el MEG representa una función de probabilidad condicional con un número finito de parámetros, contrario a los modelos econométricos tradicionales que utilizan una función de probabilidad incondicional, la cual es descrita por un número infinito de parámetros.

Esta reducción supone que el MEG puede aproximarse por la factorización de una secuencia de variables aleatorias distribuidas 
idénticamente (Hendry, 1983). Lo anterior se hace mediante la descomposición de la función conjunta de densidad del producto, las funciones de densidad condicional y la función marginal (Spanos, 1986; Galindo, 1995). Esto es válido sólo en el caso de que las variables aleatorias que generan una muestra aleatoria sean independientes y distribuidas idénticamente y que se cumpla la condición de exogeneidad débil en algunas de estas variables. Para convertir el PGI en un MEG, éste puede representarse como una función la cual transforma un conjunto de variables condicionadas a un determinado conjunto de información en un modelo que determina las variables endógenas en función de las variables exógenas y margina las variables no relevantes (Galindo, 1995; Spanos, 1986):

(5) $f\left(w_{i t} \mid \Phi\right)=f\left(y_{t}, z_{i t}, x_{i t} \mid \Phi\right)=\pi\left(y_{t}\left|y_{t-i-1} z_{i t}, x_{i t}\right| \Phi_{1}\right) \pi\left(z_{j t}\left|x_{i t}\right| \Phi_{2}\right)=\pi\left(y_{t} \mid \Omega, \lambda\right)$ donde:

$\mathrm{w}_{\mathrm{it}}$ representa las variables aleatorias;

$\Phi$ es el espacio paramétrico del PGI;

$\mathrm{y}_{\mathrm{t}}$ son las variables endógenas;

$\mathrm{z}_{\text {it }}$ son las variables exógenas;

$\mathrm{x}_{\mathrm{it}}$ son otras variables a considerar;

$\Omega$ es el conjunto de información disponible.

$\lambda$ es la transformación de $\Phi$ necesaria para que se de la factorización y, por ende, el proceso de marginalización y distribución condicional. En este caso los parámetros de $\Phi$ se mantienen constantes y son ortogonales a $\Omega$.

Por lo tanto, el comportamiento de $\lambda$ es relevante para aceptar que las variables en $\mathrm{z}_{\mathrm{it}}$ son exógenas. Esto se considera una respuesta a la crítica de Lucas, ya que los parámetros se mantienen constantes a través del tiempo (Charemza y Deadman, 1992).

El primer término de la derecha de (5) indica que se puede obtener una distribución condicional del PGI con respecto a un subconjunto de variables, mientras que el segundo supone que es posible aproximar esta función de probabilidad mediante una distri- 
bución condicional y su función marginal correspondiente. Lo anterior implica que las variables endógenas son condicionadas por las variables exógenas (Granger, 1990).

La utilización de las distribuciones de probabilidad y su marginalización permite al espacio paramétrico convertirse de un conjunto de parámetros desconocidos a los coeficientes estimables de $\lambda$. Cabe la posibilidad de que los $\lambda$ 's no sean necesariamente constantes, sin embargo, la distribución de probabilidad permite aislar la parte sistemática de aquella no sistemática y permite caracterizar el proceso estocástico como una combinación del conjunto de parámetros constantes y del término de error (Galindo, 1995). De esta manera, el modelo de probabilidad condicional conduce a varios métodos de estimación particulares en función de los supuestos adicionales que se establecen de acuerdo al comportamiento probabilístico de las series. Entre estos métodos se encuentran el método gaussiano, el de mínimos cuadrados ordinario y el dinámico lineal, entre otros.

El modelo de probabilidad condicional puede representarse como uno de regresión lineal que incluya los supuestos de linealidad y normalidad (Spanos, 1986).

(6) $y=s_{t}+e_{t} ; s_{t}=E\left(y_{t} \mid \Omega\right)$

En esta ecuación $\mathrm{s}_{\mathrm{t}}$ es la parte sistemática del proceso estocástico $\mathrm{y} \mathrm{e}_{\mathrm{t}}$ es la innovación, representando la parte no sistemática del proceso. En este caso, tanto $\mathrm{s}_{\mathrm{t}}$ como $\mathrm{e}_{\mathrm{t}}$ son definidas de acuerdo a un conjunto de información $(\Omega)$. El término de error $\left(\mathrm{e}_{\mathrm{t}}\right)$ incorpora la parte no modelada del proceso estocástico y debe considerarse como una entidad derivada, en lugar de una entidad autónoma como lo hace la econometría tradicional. Por lo que, $\mathrm{e}_{\mathrm{t}}$ es un resultado de la satisfacción de ciertos criterios preestablecidos en la modelación y tiene las siguientes propiedades:

(7) $\quad E\left(s_{t} e_{t}\right)=0$

(8) $\quad E\left(e_{t}\right)=0$

(9) $\quad E\left(e_{t}^{2}\right)=\sigma^{2}$

(10) $E\left(e_{t} e_{t-1}\right)=0$ 
Cuando hay una simplificación excesiva, para la econometría tradicional las series son no aleatorias por lo que es el término de error el que incluye las propiedades estadísticas. Para la econometría moderna, las mismas series contienen determinadas propiedades estocásticas. ${ }^{3}$ Por lo que, en la econometría moderna, lo que se analiza es que la media y la varianza de las series se mantengan estables a través del tiempo. Lo anterior permite hacer uso del teorema de Wold el cual señala que una serie estacionaria puede representarse como la suma de un componente determinístico y uno indeterminado (Cuthbertson, Hall y Taylor, 1992). Además, se hace énfasis en el uso de propiedades que tienden a ser insesgadas en muestras pequeñas. Es decir, el uso de series económicas estacionarias y las propiedades de cointegración.

Por lo que, la econometría moderna define un modelo general el cual permite, dado unos supuestos, el uso de diversos métodos econométricos aplicados a series económicas sin tener que suponer el teorema de especificación correcta de la econometría tradicional (Galindo, 1995; Spanos, 1986). Dado que el modelo de regresión lineal se define como:

$$
\text { (11) } Y_{t}=\beta^{\prime} X_{t}+\varepsilon_{t}
$$

se deben satisfacer los siguientes supuestos (Spanos, 1986; Galindo, 1995):

1. La parte sistemática del fenómeno de estudio puede modelarse como una función de probabilidad condicional:

$$
s_{t}=E\left(Y_{t} \mid \Omega\right)=\beta^{\prime} X_{t}
$$

por lo que no existe información adicional en $\mathrm{X}_{\mathrm{t}}$ que explique el comportamiento de $\mathrm{E}_{\mathrm{t}}$;

2. Los parámetros de interés $\left(\Phi_{1}\right)$ son $\beta$ y $\sigma^{2}$. Los mismos son definidos mediante el procedimiento de estimación y señalan que una serie económica puede estar caracterizada, en términos estadísticos, a través de su media y varianza. Ambas deben permanecer constantes a través del tiempo. Esto se estudia en 
la literatura econométrica moderna, mediante el análisis del orden de integración de las series y la existencia de raíces unitarias en el proceso autorregresivo de la serie. De esta manera se define un proceso como no estacionario cuando los momentos de primer y segundo orden son función del tiempo. Un proceso estacionario es uno en el que el comportamiento de los momentos de primer y segundo orden son finitos e independientes del tiempo. En este caso, la asociación entre sus diferentes realizaciones depende solamente de la distancia y, no de la posición del tiempo. Un proceso estocástico es estacionario si:

(13) $E\left(Y_{t}\right)=\bar{Y} \forall t$

(14) $\operatorname{Var}\left(Y_{t}\right)<\infty \forall t$

(15) $\operatorname{Cov}\left(Y_{t} Y_{t+k}\right)=E\left[\left(Y_{t}-\bar{Y}\right)\left(Y_{t-k}-\bar{Y}\right)\right]$

3. $\mathrm{X}_{\mathrm{t}}$ es exógena débil respecto a $\Phi_{1}$. Quiere decir que los parámetros son invariantes en el tiempo, ya que las variables exógenas del modelo no contienen información adicional que modifique el valor de los parámetros estimados (Galindo, 1995);

4. El modelo a estimar no debe incluir restricciones sobre $\Phi_{1}$ las cuales no se encuentren fundamentadas por la teoría económica ni por el conjunto de información disponible;

5. Las variables a utilizarse no son perfectamente colineales. Es decir, que el rango de $\mathrm{X}_{\mathrm{t}}=\mathrm{k}$ para toda $\mathrm{N}>\mathrm{k}$ donde $\mathrm{N}$ es el tamaño de la muestra y $\mathrm{k}$ el número de parámetros;

6. Las series estocásticas pueden ser definidas como normales e idénticamente distribuidas con media cero y varianza constante. Es decir:

$$
\begin{aligned}
& D\left(Y_{t} \mid X_{t} ; \varphi\right) \\
& E\left(Y_{t} \mid X_{t}=x_{t}\right)=\beta^{\prime} X_{t} \\
& \operatorname{Cov}\left(Y_{t} \mid X_{t}=x_{t}\right)=\sigma^{2}
\end{aligned}
$$

En este caso el modelo probabilístico se define para una distribución normal como (Spanos 1986; Galindo, 1995):

(19) $\psi\left(D Y_{t} \mid X_{t} ; \varphi\right)=\left\{\frac{1}{\sigma}\left[2 \pi \frac{1}{2}\left(\exp \left(-\frac{1}{2} \sigma 2\right)\left(Y_{t}-\beta^{\prime} X_{t}\right)^{\prime}\left(Y_{t}-\beta^{\prime} X_{t}\right)\right)\right]\right\}$; 
7. Los parámetros a estimar son invariantes en el tiempo, lo que permite que las series económicas puedan ser representadas por un conjunto finito de parámetros. Este supuesto está estrechamente vinculado al supuesto (4);

8. $\mathrm{Y}_{\mathrm{t}}$ es una muestra independiente obtenida de forma secuencial en donde se considera válido el modelo de probabilidad condicional dado por (16).

El análisis de validez de estos supuestos se realiza mediante las pruebas de especificación. Estas pruebas se concentran, básicamente, en el análisis del término de error o proceso de innovación, cuyo comportamiento resulta de satisfacer ciertos criterios preestablecidos en el proceso de modelación (Galindo, 1995). Sin embargo, desde el punto de vista metodológico, hay que hacer una distinción entre las pruebas de especificación de un modelo y los criterios de selección o pruebas sobre un modelo econométrico final (Spanos, 1986). Esto es independiente de que las pruebas econométricas puedan ser similares en ambos casos.

\section{Pruebas de diagnóstico}

En la econometría tradicional se señala que los modelos de regresión son propensos a diversos errores posibles en cuanto a la inclusión u omisión de variables irrelevantes y de variables relevantes. Esto se refleja en problemas como los de autocorrelación y heterocedasticidad. Cualquier patrón inusual de los residuos es de suma preocupación en la econometría tradicional. Por lo tanto, es esencial corroborar si un modelo, el cual se asumió de antemano que está especificado correctamente, tiene problemas de especificación. Para esto, se realiza un diagnóstico de regresión utilizando la prueba de los residuales studentizados o la prueba DFBETAS. También se realiza una prueba de diagnóstico para el error de medición cuando se considera que el término de error está correlacionado con otra variable. En este caso, como se parte de un modelo que se especificó como correcto, se comienza de lo específico a cualquier otra cosa con tal de que los errores tengan el comportamiento esperado. 
En la econometría moderna, las pruebas de diagnósticos pueden definirse como aquellas que se realizan en el modelo econométrico final para asegurarse que las transformaciones y reparametrizaciones del modelo no fueron obtenidas por el supuesto de especificación correcta. El modelo estadístico general se utiliza como marco general para realizar la selección del modelo econométrico final. Este proceso se compone de diversas transformaciones y reparametrizaciones del MEG y se conoce como el método de lo general a lo específico (Charemeza y Deadman, 1992). Es decir, se comienza con un modelo sobreparametrizado, el cual se reparametriza en variables casi ortogonales, las cuales pueden interpretarse de manera teórica. Luego, se hace la evaluación del término de error.

Los criterios para aceptar un modelo econométrico como una aproximación adecuada del PGI se basan en las pruebas de diagnóstico presentadas en el Cuadro 1 (Maddala, 1996; Galindo, 1995, Spanos, 1986): ${ }^{4}$

\section{Cuadro 1}

Pruebas de diagnóstico utilizadas en la econometría moderna

\begin{tabular}{|l|l|l|}
\hline \multicolumn{1}{|c|}{ Criterios } & \multicolumn{1}{|c|}{ Prueba(s) general(es) } & \multicolumn{1}{|c|}{ Prueba(s) particular(es) } \\
\hline 1. Coherencia con los datos & $\begin{array}{l}\text { Coeficiente de determinación } \\
\text { Autocorrelación }\end{array}$ & $\begin{array}{l}\mathrm{R}^{2} \\
\text { Durbin Watson, H-Durbin, } \\
\text { LjunBox, Multiplicadores } \\
\text { de Lagrange, Box-Pierce. } \\
\text { White, Ramsey-Reset, Glesjer, } \\
\text { Goldfeld Quant, Breush-Pagan. }\end{array}$ \\
\hline 2. Exogeneidad & Asuman & $\begin{array}{l}\text { Normalidad } \\
\text { Cambio estructural }\end{array}$ \\
\hline 3. Modelo admisible & $\begin{array}{l}\text { Jarque-Bera } \\
\text { CUSUM, CUSUMQ, Chow, } \\
\text { Chow predictiva }\end{array}$ \\
\hline 4. Restricciones válidas & Teoría económica & General a lo específico \\
\hline 5. Teoría económica & Valor de coeficientes & Prueba J \\
\hline 6. Englobamiento & Pruebas de varianza & \\
\hline
\end{tabular}

El primer criterio señala que el modelo debe producir adecuadamente el comportamiento de los datos. Quiere decir que la varianza del modelo debe representar una proporción reducida en referencia a la parte explicada del conjunto de variables (Galindo, 1995). 
También se exige que el modelo no tenga autocorrelación ni heterocedasticidad. Esto se da por tres razones principales:

1. Estas dos condiciones representan un indicio de que aún existe información que no ha sido explicada por las variables ya incluidas;

2. Esto implica una nueva búsqueda de una especificación más general;

3. Éstas representan factores potenciales que nos pueden alejar del cálculo de estimadores lineales insesgados con varianza mínima, a excepción del método CONFACT el cual permite obtener estimaciones más eficientes aprovechando la presencia de autocorrelación.

Un comportamiento o patrón sistemático del término de error puede analizarse en los siguientes dos niveles:

1. La condición débil, en la cual se prueba la presencia de autocorrelación;

2. La condición fuerte, en la cual se analiza si el término de error es predecible por su comportamiento pasado o de las otras variables en el modelo, o si es un ruido blanco.

El segundo criterio señala que si se cumple con el supuesto de exogeneidad, el proceso de probabilidad condicional realizado tiene validez. Por lo que, el conjunto de parámetros de interés contiene toda la información relevante del modelo y al ignorar $\Phi_{2}$ no se pierde información significativa. En este caso, los estimadores de MCO son consistentes ya que no existe relación entre las variables independientes y el término de error. Como la condición de exogeneidad implica estabilidad en los parámetros ante cambios en política económica, representa una solución a la crítica de Lucas (Hendry, 1997). La constancia de los parámetros es consistente con el supuesto de exogeneidad débil, el cual es fundamental para que se cumpla con el de exogeneidad fuerte (no causalidad en el sentido de Granger) y el de superexogeneidad (pruebas de cambio estructural explicas en el tercer criterio). 
Dados los primeros dos criterios, el modelo se encuentra en condiciones de realizar las proyecciones y simulaciones necesarias. De este modo, el tercer criterio establece que el modelo es admisible en cuanto a los datos suministrados.

Según el cuarto criterio, las restricciones en los parámetros deben ser consistentes con las impuestas por el modelo inicial. Además, debe guardar consistencia con la teoría económica, según lo indica el quinto criterio.

El modelo econométrico final debe tener la facultad de englobar o explicar resultados econométricos previos. Atendiendo el concepto de dominación de varianza y a la prueba j. Sin embargo, la instrumentación de esta prueba es muy compleja, ya que los modelos econométricos tienden a diferir por razones diversas, entre éstas:

- Distintas bases de datos;

- Uso de series con o sin estacionalidad;

- Diferentes transformaciones de los datos;

- Diversas formas funcionales;

- Distintas formas dinámicas;

- Diferentes métodos de estimación;

- Diversas concepciones sobre las pruebas de diagnósticos.

No obstante, el criterio de englobamiento incluye el criterio de Ockhan el cual establece que en igualdad de condiciones debe escogerse el modelo más simple (Galindo, 1995). Una vez el modelo econométrico final satisface las condiciones anteriores se considera como uno congruente.

\section{Conclusiones}

Las proyecciones de las principales variables macroeconómicas diversas en Puerto Rico se basan en el uso de un sistema de ecuaciones simultáneas. Esto modelos han sido muy criticados ya que presentan los mismos problemas que los modelos estructurales en la década de 1970, los cuales provocaron una revisión profunda de las bases de la econometría y sus métodos de estimación. 
Esto no se ha realizado hasta el momento en la Isla y se está, por tanto, muy rezagado.

Esta revisión se basó en dos puntos principales: la metodología de la econometría moderna y la patología de los estimadores. Un cambio fundamental es que se presume que la econometría funciona con series estocásticas que se generan por un proceso generador de información. En este caso, el término de error es sólo una entidad derivada de las propiedades estadísticas de las series, en lugar de un término independiente que permite incluir ciertas propiedades estocásticas a un modelo.

Lo anterior implicó el uso de las propiedades de las series como un conjunto de información y que el modelo final debe satisfacer ciertos criterios para ser uno congruente. Esta metodología enfatiza las propiedades estadísticas que validan el uso de las estimaciones por mínimos cuadrados ordinarios, al incluir un conjunto de pruebas estadísticas que convaliden el uso de ciertos tipos de estimación econométrica.

Se recomienda el diseño de modelos nuevos, altamente basados en la metodología econométrica moderna, para analizar y proyectar la actividad económica en Puerto Rico, ya que aspectos como los antes mencionados son fundamentales, no llevar dicho análisis correctamente puede conducir a conclusiones erróneas en términos de la toma de decisiones. Este planteamiento es clave en cualquier modelo que utilice series de tiempo. 


\section{Notas}

${ }^{1}$ En este caso, la validez de un método econométrico se define en función a la evidencia empírica y al propósito específico para la cual se utilice.

${ }^{2}$ Heterogeneidad en el tiempo implica que la distribución de probabilidad conjunta depende del tiempo (Galindo, 1995; Hendry, 1997; Spanos, 1986).

${ }^{3}$ Esto puede verse al analizar el sesgo de los estimadores obtenidos por mínimos cuadrados ordinarios (MCO). Puede hacer referencia a Maddala (1996) y Spanos(1986).

4 Dichos criterios no son presentados en orden de importancia. 
UNA NUEVA PROPUESTA PARA MODELAR Y PROYECTAR LA ACTIVIDAD ECONÓMICA...

\section{Bibliografía}

Banerjee, A.J.; Dolado, J.; Galbraith, J.W. y Hendry, D.F. (1993). CoIntegration, Error-Correction, and the Econometric Analysis of NonStationary Data. Reino Unido, Oxford University Press.

Clements, M.P. (1995). "Rationality and the Role of Judgment in Macroeconomic Forecasting." The Economic Journal, Núm. 429, Vol. 105, pp. 410-430.

Cuthbertson, K.; Hall, S.G. y Taylor, M.P. (1992). "Applied Econometric Techniques". Reino Unido, Pearson Higher Education.

Charemza, W. y Deadman, D.F. (1993). "New Directions in Econometric

Practice." General to Specific Modeling, Cointegration and Vector Autorregresion, Editor Edward Elgar. Publicación limitada, Reino Unido, Cambridge University Press.

Eatwell J., Milligate M. y Newman, P. (1990). Time Series and Statistics. New York, W.W. Norton.

Enders, W. (1995). Applied Econometrics Time Series. New York, Wiley.

Ericsson, N.R. y Irons, J.S. (1994). “Testing Exogeneity.” Reino Unido, Oxford University Press.

Galindo, L.M. (1997). "El concepto de exogeneidad en la econometría moderna." Investigación económica, Núm. 220, pp. 97-111.

.(1995). "La metodología econométrica moderna: una versión aplicada”. Economía moderna aplicada: Cuadernos de trabajo, Núm. 18. UACPYP, CCH, UNAM, pp. 1-30.

Galindo, L.M. y Cardero, M.E. (1998), "Modelo de vectores autorregresivos con cointegración para la economía mexicana: 1980-1996.” Economía Mexicana, Vol. VI, Núm. 2, pp. 223-246.

Gilbert, C.L. (1990). "Professor Hendry's Econometric Methology". In Modelling Economic Series, Ed. C.W.J. Granger. Reino Unido, Oxford University Press.

Granger, C.W. y Newbold, P. (1974) "Spurious Regressions in Econometrics.” Journal of Econometrics, Vol. 2.

Hendry, D.F. (1997). "The Econometrics of Economic Forecasting." The Economic Journal, Vol. 47, Núm. 444, pp. 1311-1656. Press. (1995). Dynamic Econometric. Reino Unido, Oxford University

Johansen, S. (1988). "Statistical Analysis of Cointegration Vectors." Journal of Economic Dynamic and Control, Núm. 12, pp. 231-54. 
Junta de Planificación de Puerto Rico (1989). Modelos económicos: su estructura y usos. Area de Planificación Económica y Social, Subprograma de Análisis Social, Modelos y Proyecciones.

King, R.G y Plosser, C. (1984). "Money, Credit and Prices in Real Bussines Cycles." American Economic Review, Vol. 74.

Lucas, R.E. y Sargent, T.J. (1975). "After Keynesian Macroeconomics." Rational Expectations and Econometric Practice. Editado por Robert Lucas y Thomas Sargent. Vol.1. The University of Minnesota Press, Minneapolis, 1982.

Lucas, R.E. (1972). "Expectations and the Neutrality of Money." Journal of Economic Theory, Núm. 4, pp. 103-124.

Maddala, G.S. (1996). Introducción a la Econometría. Segunda Edición. España, Prentice Hall.

Maddala, G.S. y Kim, I.M. (1998). Unit Roots, Cointegration and Structural Change. Primera Edición. Reino Unido, Cambridge University Press.

Pankratz, A. (1995). Forecasting With Univariate Box-Jenkins Models: Concepts and Cases. New York, John Wiley \& Sons, Inc.

Sims, C. y Zha, T.A. (1998). "Does Monetary Policy Generates Recessions?" Documento de trabajo del Banco de la Reserva Federal de Atlanta.

Sims, C. (1987). "Comment." Journal of Business and Economics Statistics, Vol. 5, pp. 443-449.

(1975). "Exogenity and Causal Ordering in Macroeconomics Models in New Methods in Bussiness Cycle Research." Trabajo presentado en una conferencia del Banco de la Reserva Federal de Minneapolis.

. (1980). "Macroeconomics and Reality." Econométrica, Vol. 48, Núm. 1, pp. 1 - 48.

(1986). "Are Forecasting Models Usable for Policy Analysis?" Reserve Bank of Minneapolis Quaterly Review, pp. 3-16.

Spanos, A. (1986). Statistical Foundations of Econometric Modeling. Reino Unido, Cambridge University Press. 